\title{
The Active Atlas: Combining 3D Anatomical Models with Texture Detectors
}

\author{
Yuncong Chen ${ }^{1}$, Lauren McElvain ${ }^{2}$, Alex Tolpygo ${ }^{3}$, Daniel Ferrante ${ }^{3}$, \\ Harvey Karten ${ }^{2}$, Partha Mitra $^{3}$, David Kleinfeld ${ }^{2}$, Yoav Freund ${ }^{1}$ \\ 1 Department of Computer Science and Engineering, University of California, San \\ Diego, La Jolla, USA \\ \{yuncong, yoav\}@ucsd.edu \\ 2 Department of Physics, Univerity of California, San Diego, La Jolla, USA \\ 3 Cold Spring Harbor Laboratory, Cold Spring Harbor, New York, USA
}

\begin{abstract}
While modern imaging technologies such as fMRI have opened exciting possibilities for studying the brain in vivo, histological sections remain the best way to study brain anatomy at the level of neurons. The procedure for building histological atlas changed little since 1909 and identifying brain regions is a still a labor intensive process performed only by experienced neuroanatomists. Existing digital atlases such as the Allen Reference Atlas are constructed using downsampled images and can not reliably map low-contrast parts such as brainstem, which is usually annotated based on high-resolution cellular texture.

We have developed a digital atlas methodology that combines information about the $3 \mathrm{D}$ organization and the detailed texture of different structures. Using the methodology we developed an atlas for the mouse brainstem, a region for which there are currently no good atlases. Our atlas is "active" in that it can be used to automatically align a histological stack to the atlas, thus reducing the work of the neuroanatomist.
\end{abstract}

\section{Introduction}

Pioneered by Korbinian Brodmannn in 1909 [3, the classical approach to mapping distinct brain regions is based on visually recognizing the cellular textures (cytoarchitecture) from images of sections of a brain. Several paper atlases have been created in this way for the brains of different species [10].

The primary methods for expert annotation of brain regions have changed little since then. It still is a labor intensive process performed only by the most experienced neuroanatomists. In this paper we propose a machine learning approach for atlas construction that uses automated texture recognition to immitate human pattern recognition in the annotation task.

There exist several section-based digital atlases that were constructed using automated registration algorithms. The best known is the Allen Reference Atlas for mouse 1, 4, 6], which is based on downsampled images of $50 \mu \mathrm{m}$ per pixel. At this resolution, registration can be performed by maximizing intensity similarity using metrics such as correlation and mutual information. 


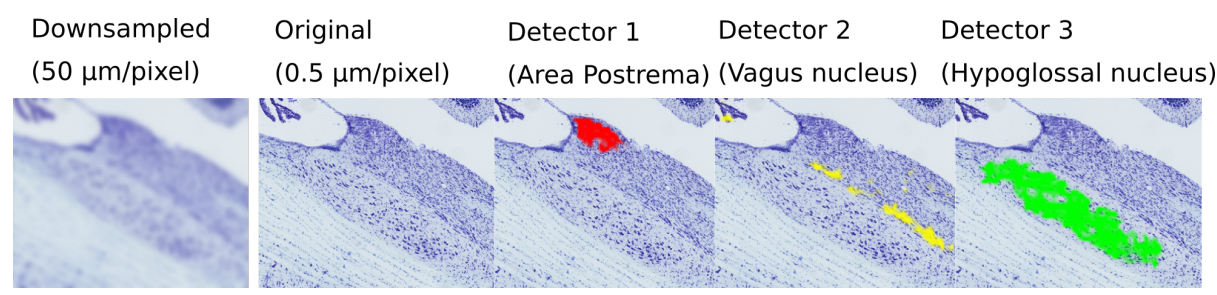

Fig. 1. A demonstration of the limitation of reduced resolution brain images. The "Original" image was taken at $0.5 \mu \mathrm{m} /$ pixel. "Detector $1,2,3$ " represent the detection of three brain structures based on texture by the trained classifiers. The "Downsampled" image lacks the high-resolution details needed to distinguish the structure. (Best viewed in color)

The problem is that at this resolution the information on cellular texture is discarded, which results in poor localization in regions that lack high contrast boundaries (see Figure 1). In this work we focus on the mouse brainstem, a part that has numerous cytoarchitecturally identifiable nuclei but is relatively homogeneous at low resolution. To overcome this limitation we have developed the active atlas, a texture-based atlas that operates on the full-resolution images and uses texture classifiers to differentiate structures not identifiable at low resolution. This distinguishes our approach from both the Allen atlas and those based on MRI or optical volumes 8, $8,11,13$.

The contributions of this work are:

- Detection of cytoarchitectural textures visible only at high resolution.

- Identification of discrete structures in addition to overall registration.

- Characterization of the positional variability of brain structures.

- Use of iterative refinement to reduce human annotation effort.

The paper is organized as follows. Section 2 describes the procedure for building an active atlas. Section 3 presents evaluation results that demonstrate the confidence of registration and accuracy of texture detection.

\section{The Active Atlas}

The active atlas has two components:

1. Anatomical model: stores for each of 28 structures in the brainstem, the position statistics and probabilistic shape.

2. Texture classifiers: predict the probability that a given image patch corresponds to a particular structure.

The construction of the atlas is iterative, starting with an initialization step that required significant human labor, followed by refinement steps which require little or no expert labor (see Figure 2). In our case, the initial step was to annotate three stacks of images, which required 30 hours of work of an experienced neuroanatomist. From these annotated stacks, an initial anatomical 


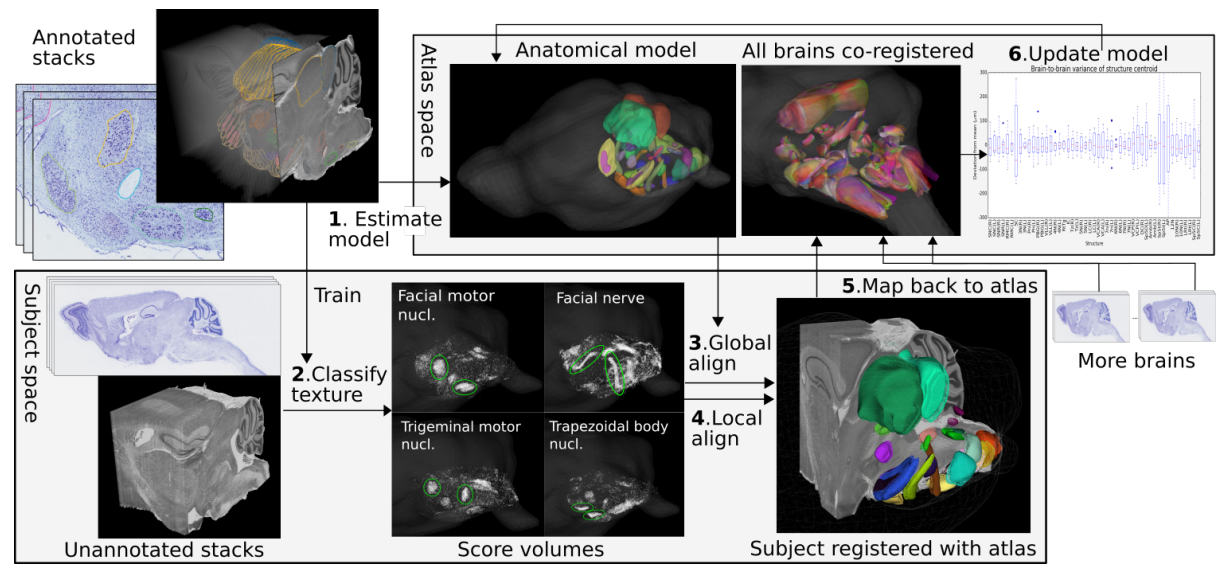

Fig. 2. Incremental atlas building workflow

model and a set of texture classifiers were constructed. The refinement then uses nine additional stacks that were not annotated. These stacks were aligned to the initial atlas and the information from this alignment was used to refine the atlas and to estimate the variability from brain to brain. Below we provide more details on each step.

2.1 Preprocessing. Our dataset consist of sagittal brain sections from twelve mice of identical strain and age. The $20 \mu \mathrm{m}$ sections are mounted with a tapetransfer system [12 to ensure minimal distortion. Each specimen gives roughly 400 sections, stained with Nissl and scanned at $0.5 \mu \mathrm{m}$ resolution, demonstrating clear cytoarchitectonic features. The sections of each brain are registered via inplane correlation-maximizing rigid transforms and stacked to reconstruct a 3D volume. The sufficiency of rigid transforms is proved by the smooth structure boundaries on virtual coronal sections of reconstructed volumes.

2.2 Estimation of Anatomical Model. Model estimation takes as input a current model (initially null) and a set of manually or automatically annotated brains. From each annotated brain one can collect an aligned contour set for each structure (Figure 3b), which can be converted into a 3D mesh or volume (Figure 3.). Based on them we derive the average centroid position and the average shape of each structure, which constitute a refined model.

2.2.1 Position Estimation. All brains are co-registered using the method described in Section 2.4. Centroid positions of the same structures in the common space are averaged over all brains. Those of paired structures are further adjusted to ensure symmetry of left and right hemispheres. The covariance matrices of centroid positions are also computed. They quantify brain variability and are used as structure-specific constrains for aligning future data.

2.2.2 Shape Estimation. All meshes of the same structure are aligned using Iterative Closest Point algorithm 2] (Figure 3d) and converted to aligned volumes. 

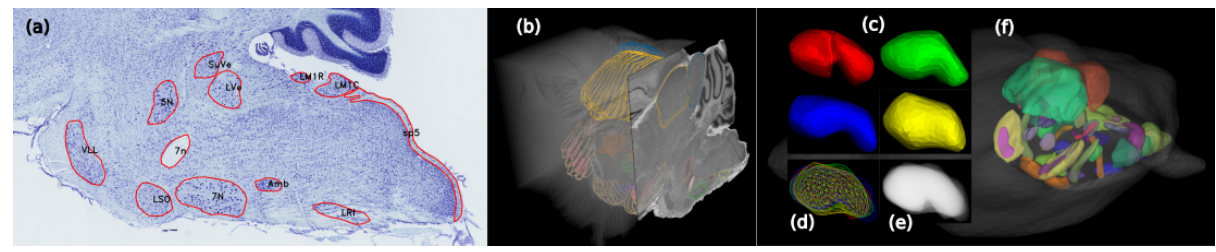

Fig. 3. (a) Structure boundaries drawn by an expert (b) Aligned contour series in 3D (c) Facial motor nucleus from both hemispheres of different brains (d) Meshes aligned (e) Probabilistic average shape (f) Anatomical model with 28 structures

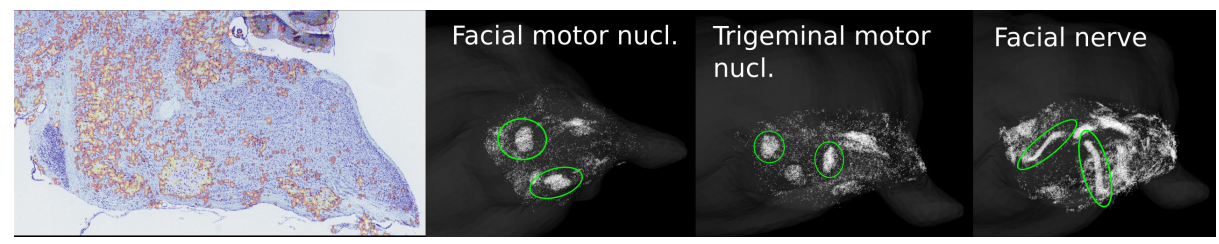

Fig. 4. (a) An example score map for facial motor nucleus (b) Stacking 2D score maps forms a $3 \mathrm{D}$ score volume. (c,d) Score volumes for other structures.

The average shape as a probabilistic volume is then computed by voxel-voting (Figure 3e).

Combining average shapes with average centroid positions, we obtain a probabilistic atlas volume $\mathbf{A}$ where $\mathbf{A}(\mathbf{p})$ denotes the 28 -dimensional probability vector at location $\mathbf{p}$.

2.3 Learning Texture Classifiers. We train texture classifiers to differentiate a structure from its immediate surrounding region. We found that this gives better results than training against the entire background. The probable reason is that the anatomical model eliminates most of the uncertainty in gross positions, allowing the texture classifiers to focus on correcting small-scale error.

Image patches roughly $100 \mu \mathrm{m}$ by $100 \mu \mathrm{m}$ are used as units for classification. For each structure, a binary logistic regression classifier is trained using a positive patch set extracted from the interior of structure boundaries and a negative set extracted from the surrounding region within $50 \mu \mathrm{m}$ from the boundaries. The feature vectors encoding the patches are the 1024-dimensional output of a pre-trained deep convolutional neural network (Inception-BN 7]). Although the network was originally trained for classifying natural images, it proves effective also for classifying histology textures.

For an unannotated image, these classifiers are applied to patches with $25 \mu \mathrm{m}$ spacing, resulting in score maps for different structures. All score maps of a same structure in one stack undergo the previously computed intra-stack alignment to form a set of 3D score volumes. Each volume represents a probabilistic estimate of a particular structure's position in the reconstructed specimen (Figure 4). Denote by $\mathbf{S}(\mathbf{p})$ the vector consisting of the scores for different structures at location $\mathbf{p}$. 
2.4 Registering Atlas to Specimen. Registration is driven by maximizing the correlation at all voxels between the score vectors of the specimen volume and the probability vectors of the atlas volume. A global 3D transform first aligns the atlas roughly with the whole specimen. Affine transform is used to account for non-vertical cutting angle and scale change due to dehydration. Separate 3D translations are then applied to each structure so independent variations can be captured.

Let $\Omega$ be the domain of the atlas. For global transform, the objective to maximize is simply $F^{\text {global }}(\mathbf{L}, \mathbf{b})=\sum_{\mathbf{p} \in \Omega} \mathbf{A}(\mathbf{p}) \cdot \mathbf{S}(\mathbf{L} \mathbf{p}+\mathbf{b})$, where $\mathbf{L} \in \mathbb{R}^{3 \times 3}$ and $\mathbf{b} \in \mathbb{R}^{3}$ are respectively the linear and translation parts of the affine transform.

For the local transform of structure $k$, only the voxels inside the structure and those in a surrounding region within a $50 \mu \mathrm{m}$ radius are concerned. Denote the two sets by $\Omega_{k}^{+}$and $\Omega_{k}^{-}$respectively. The objective is

$$
F^{\text {local }}(\mathbf{t})=\sum_{\mathbf{p} \in \Omega_{k}^{+}} \mathbf{A}(\mathbf{p}) \cdot \mathbf{S}^{\prime}(\mathbf{p}+\mathbf{t})-\sum_{\mathbf{p} \in \Omega_{k}^{-}} \mathbf{A}(\mathbf{p}) \cdot \mathbf{S}^{\prime}(\mathbf{p}+\mathbf{t})-\eta \mathbf{t}^{\top} C_{k} \mathbf{t}
$$

where $\mathbf{t} \in \mathbb{R}^{3}$ is the local translation and $\mathbf{S}^{\prime}$ is the globally transformed score volume. The regularization term penalizes deviation from the mean position defined in the atlas model, where $C_{k}$ is the inverse of the position covariance matrix (see Section 2.2.1).

Optimization for both cases starts with grid search, followed by gradient descent where the learning rate is determined using Adagrad [5]. From Figure 5 one can visually verify the accuracy of registration. This registration effectively annotates new stacks for the 28 structures.
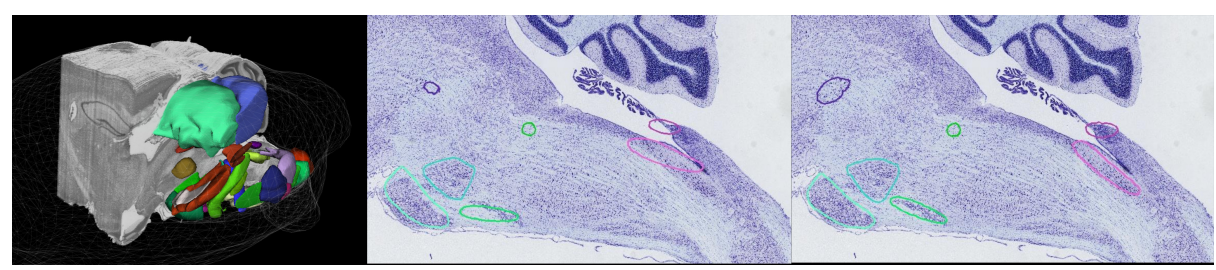

Fig. 5. (a) Reference model globally registered to the specimen. (b) Global registration. Showing the structure contours on a section. Structures are roughly aligned. (c) Local registration. Structures are aligned perfectly.

2.5 Evaluating Registration Confidence. The registration algorithm seeks a local maxima of the objective functions. We quantify the confidence of the registration by considering the height and the width of the converged local maxima. The height of the peak is normalized by considering a $z$-test relative to the variance within a sphere around the peak. The width can be computed for any direction, based on the Hessian of the $z$-score around the peak, as the distance away from peak that the $z$-score drops to 0. Figure 6 shows examples where different directions have different localization confidence. 

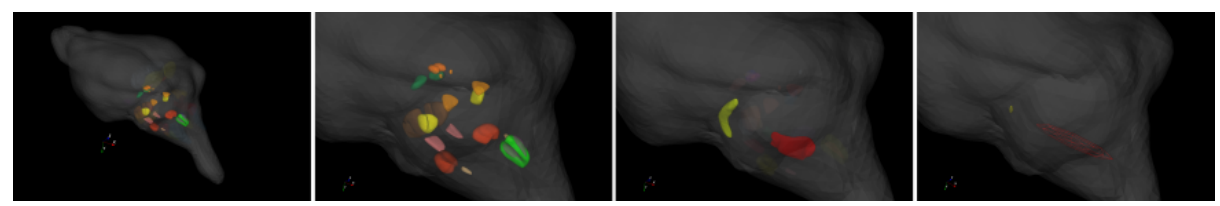

Fig. 6. (a, b) Confident structures (c) Two unconfident structures (d) Uncertainty ellipsoids. The elongated structure VLL (yellow) is uncertain only in its axial direction, while Sp5I (red) is uncertain in rostral-caudal direction because its rostral and caudal boundaries are ambiguous. (Best viewed in color)

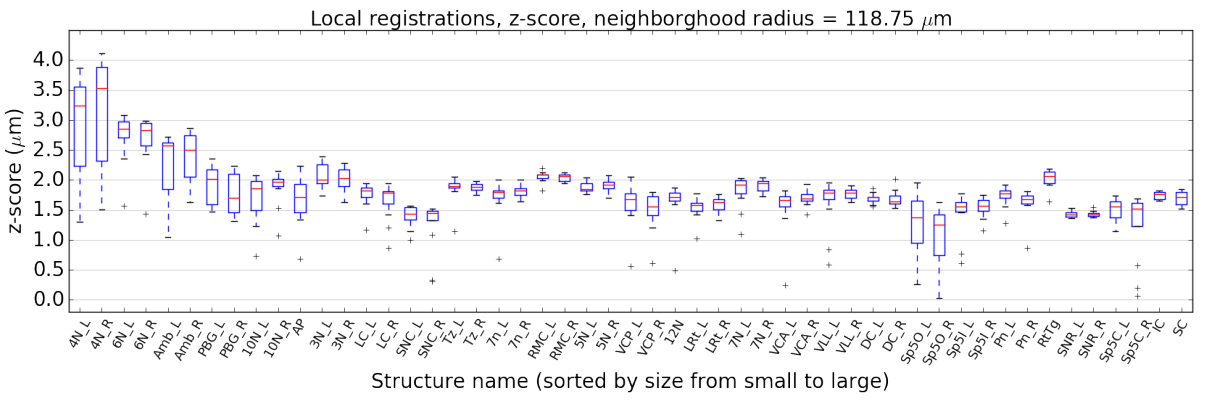

Fig. 7. Average z-scores of the local registrations of different structures.

2.6 Updating Atlas. After new brains are co-registered with the atlas, average positions and shapes for all structures are re-estimated. Additional training patches can also be collected from the automatic annotations to improve the classifiers.

\section{Results}

3.1 Confidence of Registrations. The global registrations across all specimens have an average peak z-score of 2.06 . The average peak radius is $98 \mu \mathrm{m}$ in the steepest direction and $123 \mu \mathrm{m}$ in the flattest direction. This suggests that the derived reference model captures the common anatomy of this population and matches all specimens with little space for adjustment. Figure 7 and 8 show these for the per-structure registrations. The average z-score is 1.79 and the width is between $90 \mu \mathrm{m}$ and $250 \mu \mathrm{m}$ for most structures. Generally, small structures tend to be registered more confidently than large ones. This aligns well with intuition if one considers how position shifts affect the overlap between the structure and the texture map. For a small structure, a small translation might completely eliminate any overlap, while a large structure is less sensitive.

3.2 Variability of Structure Position. Variability is captured by the amount of per-structure translation. Figure 9 shows these for different structures across all specimens. Most structures vary within 100um of the mean position defined in atlas. Some structures are particularly variable, which are also the ones whose 

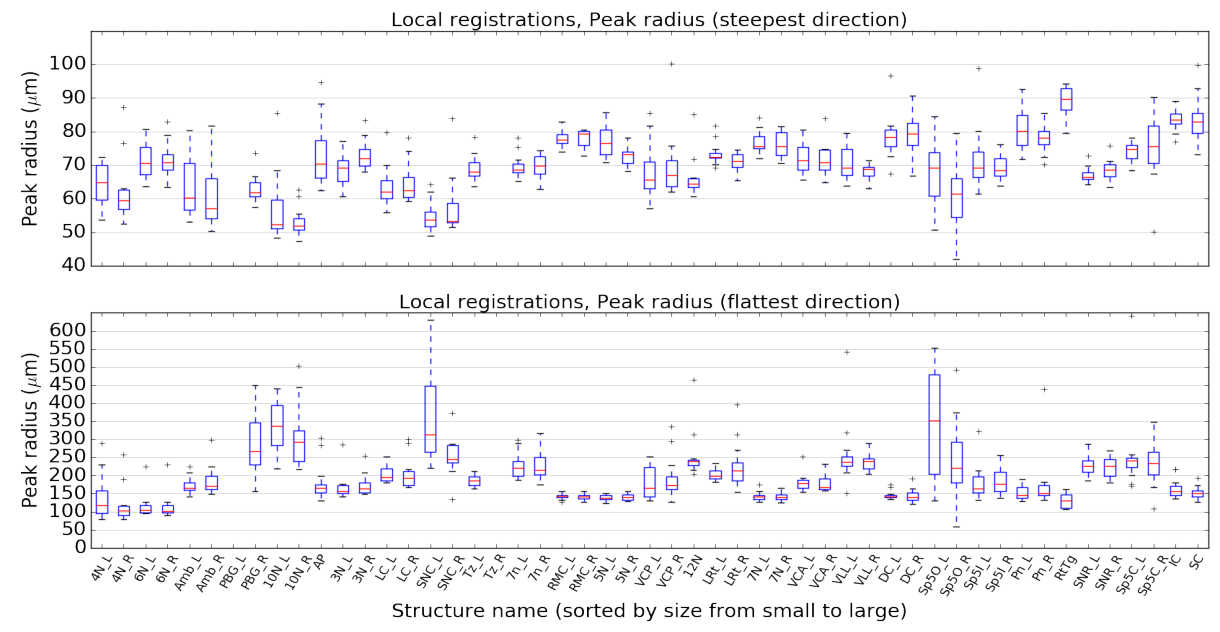

Fig. 8. Average peak width of per-structure registrations of different structures.

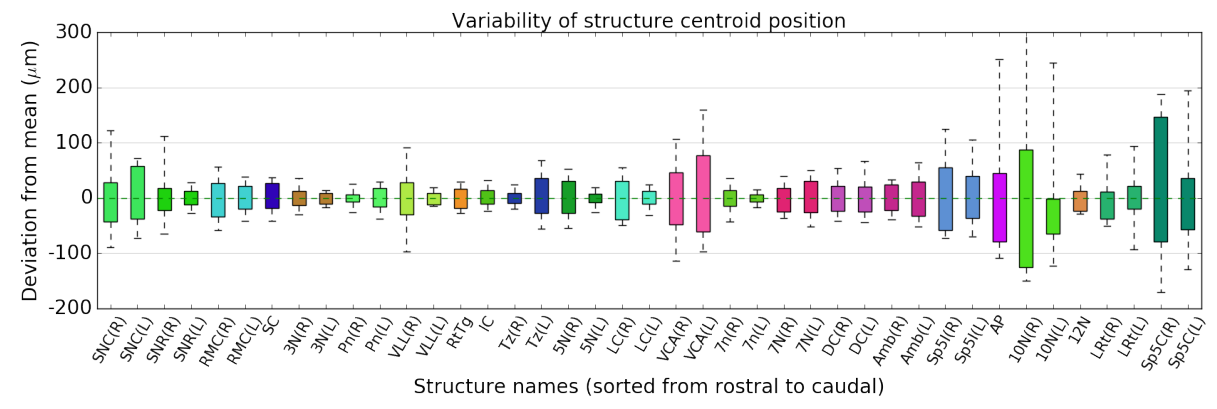

Fig. 9. Variability of centroid positions for different structures. Same color indicates the same structure in left $(\mathrm{L})$ and right $(\mathrm{R})$ hemispheres.

boundaries are difficult to define. The same structure in left and right hemispheres generally have similar variability.

3.3 Accuracy of Texture Classifiers. Figure 10 shows the test accuracy for the classification of different structures. They range from 0.7 to 0.9 with a mean of 0.79. Larger structures tend to be harder to classify possibly due to their texture being more inhomogeneus.

\section{Conclusion}

The results demonstrate a form of co-training between the anatomical model and the texture classifiers. On the one hand, registrations perform well despite the classifiers for some structures are suboptimal, due to the strong constraint by the anatomical model. On the other hand, confident detection of the characteristic 


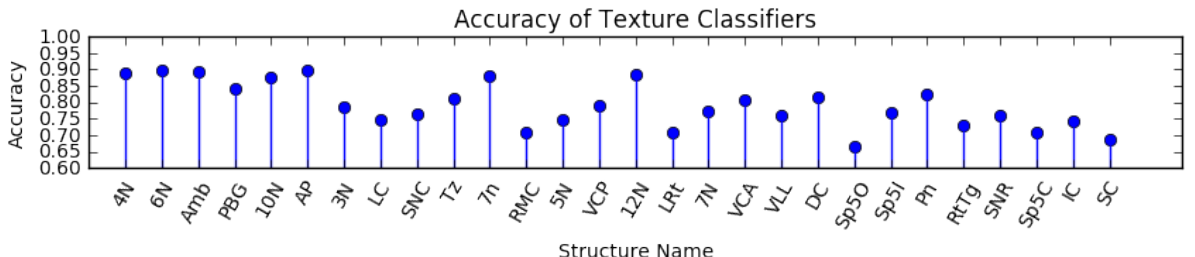

Fig. 10. Accuracy of texture classifiers. (Structures sorted by increasing size)

textures of many structures allows specimen-specific deviations from the current anatomical model to be discovered, contributing to more accurate variability. The synergy between the anatomical information and texural information is the key feature of the proposed active atlas.

\section{References}

1. Technical white paper: Allen mouse common coordinate framework. Tech. rep. (2015)

2. Besl, P.J., McKay, N.D.: Method for registration of 3-d shapes. Proc. SPIE 1611, 586-606 (1992)

3. Brodmann, K., Garey, L.: Brodmann's: Localisation in the Cerebral Cortex. Springer (2006)

4. Dong, H.: Allen Reference Atlas: a digital color brain atlas of the C57BL/6J male mouse. John Wiley \& Sons (2008)

5. Duchi, J., Hazan, E., Singer, Y.: Adaptive subgradient methods for online learning and stochastic optimization. JMLR 12(Jul), 2121-2159 (2011)

6. Fonov, V., et al.: Unbiased average age-appropriate atlases for pediatric studies. Neuroimage 54(1), 313-327 (2011)

7. Ioffe, S., Szegedy, C.: Batch normalization: Accelerating deep network training by reducing internal covariate shift. arXiv preprint arXiv:1502.03167 (2015)

8. Johnson, G.A., et al.: Waxholm space: an image-based reference for coordinating mouse brain research. Neuroimage 53(2), 365-372 (2010)

9. Mazziotta, J., Toga, A., et al.: A probabilistic atlas and reference system for the human brain: International consortium for brain mapping (icbm). Philosophical Transactions of the Royal Society of London B: Biological Sciences 356(1412), 1293-1322 (2001)

10. Paxinos, G., Franklin, K.B.: The mouse brain in stereotaxic coordinates. Gulf Professional Publishing (2004)

11. Peng, H., et al.: Brainaligner: 3 d registration atlases of drosophila brains. Nature Methods 8(6), 493-498 (2011)

12. Pinskiy, V., Jones, J., Tolpygo, A.S., Franciotti, N., Weber, K., Mitra, P.P.: Highthroughput method of whole-brain sectioning, using the tape-transfer technique. PLoS ONE 10(7) (2015)

13. Ronneberger, O., et al.: Vibe-z: a framework for $3 \mathrm{~d}$ virtual colocalization analysis in zebrafish larval brains. Nature Methods 9(7), 735-742 (2012) 